Accurate taxi time prediction is required for enabling efficient runway scheduling that can increase runway throughput and reduce taxi times and fuel consumptions on the airport surface. Currently NASA and American Airlines are jointly developing a decision-support tool called Spot and Runway Departure Advisor (SARDA) that assists airport ramp controllers to make gate pushback decisions and improve the overall efficiency of airport surface traffic. In this presentation, we propose to use Linear Optimized Sequencing (LINOS), a discrete-event fast-time simulation tool, to predict taxi times and provide the estimates to the runway scheduler in real-time airport operations. To assess its prediction accuracy, we also introduce a data-driven analytical method using machine learning techniques. These two taxi time prediction methods are evaluated with actual taxi time data obtained from the SARDA human-in-theloop (HITL) simulation for Charlotte Douglas International Airport (CLT) using various performance measurement metrics. Based on the taxi time prediction results, we also discuss how the prediction accuracy can be affected by the operational complexity at this airport and how we can improve the fasttime simulation model before implementing it with an airport scheduling algorithm in a real-time environment. 


\title{
Taxi Time Prediction at Charlotte Airport Using Fast-Time Simulation and Machine Learning Techniques
}

\author{
Hanbong Lee \\ University of California, Santa Cruz \\ NASA Ames Research Center
}

Joint Workshop for KAIA/KARI/IIAC-NASA Collaboration

Korea Aerospace Research Institute, Daejeon, Korea

Incheon International Airport, Incheon, Korea

April 5-7, 2016 


\section{Outline}

- Introduction: Aircraft taxi time prediction

- Previous research

- Surface traffic at Charlotte airport (CLT)

- Human-In-The-Loop (HITL) simulation data for CLT

- Two taxi time prediction approaches

- Data-driven analytical method using machine learning

- Fast-time air traffic simulation using LINOS

- Taxi time prediction performance comparison

- Conclusions

- Future work 


\section{Motivation}

- Aircraft taxi time prediction

- Estimate wheels-off times of departures

- Determine gate-holding times to reduce taxi times

- Assign the best gates to turnaround flights

- Accurate prediction is difficult.

- Uncertainties in airport operations

- Operational complexity

- Hard to get accurate prediction from data-driven methods

- Fast-time simulation

- Alternative taxi time prediction approach

- Can reflect operational conditions and uncertainties

- Can run in real-time operations 


\section{Objectives}

- Develop precise taxi time prediction modules

- Fast-time simulation tools like LINOS

- Data-driven analytical methods using machine learning

- Evaluate taxi time prediction performance

- Using Human-In-The-Loop (HITL) simulation data

- Comparison of prediction methods

- Integrate taxi time prediction module with DecisionSupport Tool (DST) for controllers

- Provide estimated wheels-off times of departures to a runway scheduling algorithm

- Reflect current aircraft states in real-time operations 


\section{Previous Research (1/2)}

- Queuing models for taxi-out time estimation

- Research in road travel time prediction

- Various machine learning methods

- Include linear regression models, neural networks, regression trees, $k$-nearest neighbors, and support vector machines

- Machine learning based taxi time prediction approaches

- Linear regression models

- Neural network model

- Reinforcement learning algorithms 


\section{Previous Research (2/2)}

- Fast-time simulation tools for modeling airport operations

- Test various traffic scenarios at airports and airspace

- Forecast upcoming traffic status quickly, including expected takeoff times of departures

- Microscopic simulation tools reflecting individual flight movements

- Based on discrete-event simulation models

- Node-link networks representing airport layouts

- Can predict 4D aircraft trajectories 
- LINear Optimized Sequencing (LINOS)

- A fast-time simulation tool developed by American Airlines for surface traffic modeling

- Node-link network accounting for aircraft capability and operational conditions

- LINOS can run continuously with current aircraft status and update its predictions whenever events occur.

- LINOS input data

- Basic flight plan information

- Latest gate departure times

- Estimated landing times

- Current positions of taxiing aircraft 


\section{Surface Traffic at Charlotte Airport (CLT)}

- Runway configuration

- (18R, 23 | 18C, 18L)

- South-flow traffic

- Ramp area controlled by American Airlines

- Single lane bottleneck

- Head-on conflicts possible

- Alternative M-C taxi route for arrivals, if permitted by ATC

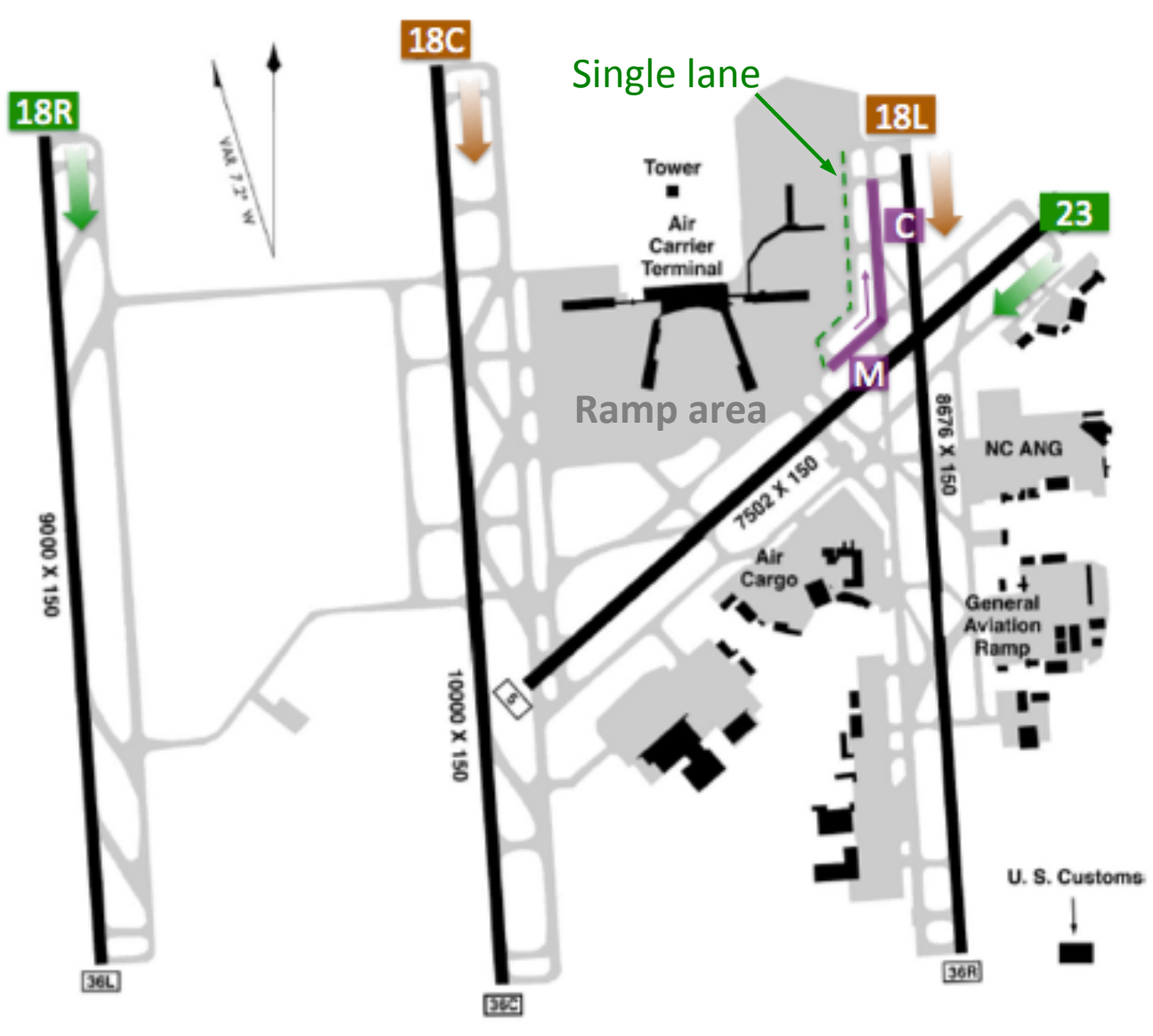




\section{Spot And Runway Departure Advisor (SARDA) Scheduler}

- Human-In-The-Loop (HITL) Simulation for CLT

- Conducted by NASA Ames and American Airlines to test a new pushback decision-support tool for CLT (SARDA-CLT)

- Performed at NASA Ames FutureFlight Central facility for 3 weeks in 2014

- Departure pushback advisories for ramp controllers

- Specify whether a departure should be released or held at gate for certain duration

- Calculated after optimizing runway schedule

- Dead Reckoning (DR) method for taxi time prediction based on unimpeded taxi times 


\section{Simulation Data for CLT}

- Three-week data collected from HITL simulation for CLT

- Two one-hour long scenarios

- Based on actual traffic data from CLT (May 16, 2013)

- Departure push followed by arrival push

- Scenario 1: 96 departures \& 80 arrivals

- Scenario 2: 84 departures \& 72 arrivals

- Two types of simulation runs

- Baseline: similar to current operations

- Advisory: following pushback advisories from SARDA scheduler 


\section{Two Approaches to Predicting Taxi Times}

Data-driven analytical

approach

- Use machine learning techniques

- Based on historical data

- Need to determine variables impacting taxi time prediction

- Hard to apply to real-time operations, if available data are limited

\section{Fast-time simulation-} based approach

- Use LINOS as an air traffic simulation tool

- Node-link network modeling airport layout

- Need to consider airport specific operational constraints

- Applicable to real-time operations 


\section{Machine Learning Approach}

- Features (variables): Gate, Spot, Runway, Aircraft model, Taxi distance, Number of taxiing departures and arrivals

- Machine learning techniques used

- Linear Regression (LR)

- Support Vector Machines (SVM)

- $k$-Nearest Neighbors ( $k N N)$

- Random Forest (RF)

- Trained with Week 1 \& 2 data from HITL simulation

- By traffic scenario (Scenario 1 and 2)

- By advisory mode (Baseline and Advisory)

- Tested with Week 3 data for performance comparison 


\section{Fast-Time Simulation Approach}

- LINOS for taxi time predictions

- Node-link network model for CLT

- Operational parameters calibrated with Week 1 \& 2 data, such as separation times, pushback times, and taxi speeds

- Tested with Week 3 data for performance comparison

- Ran with static input data for each run, instead of updating airport state every 10 seconds

- Repeated the same simulations 10 times for operational uncertainties and used the average taxi time for prediction

- Considered Traffic Management Initiatives (TMIs) and Alternative taxi routes 


\section{Taxi Time Prediction Results}

- Mean taxi time difference between predicted taxi times and actual times from HITL simulation data

- Prediction approaches: small gap (<0.4 minutes)

- Dead Reckoning method based on unimpeded taxi times:

5-7 minutes shorter than actual

- Both prediction approaches showed much better prediction performance than Dead Reckoning method.

Performance Metric: Root Mean-Squared Error (Unit: minutes)

\begin{tabular}{|c|c|c|c|c|c|c|}
\hline \multirow{2}{*}{ Test Dataset } & \multicolumn{4}{|c|}{ Machine Learning Methods } & \multirow{2}{*}{ LINOS } & \multirow{2}{*}{$\begin{array}{c}\text { Dead } \\
\text { Reckoning }\end{array}$} \\
\hline & LR & SVM & $k N N$ & RF & & \\
\hline Baseline runs & 2.83 & 2.29 & 1.22 & 1.15 & 2.67 & 7.14 \\
\hline Advisory runs & 2.62 & 2.16 & 1.12 & 1.21 & 2.06 & 6.25 \\
\hline
\end{tabular}




\section{Departure Taxi Time Differences}

- Best performance in $k \mathrm{NN}$ and RF methods

- Possibly over-trained from repeated simulations

- Better prediction accuracy in Advisory cases

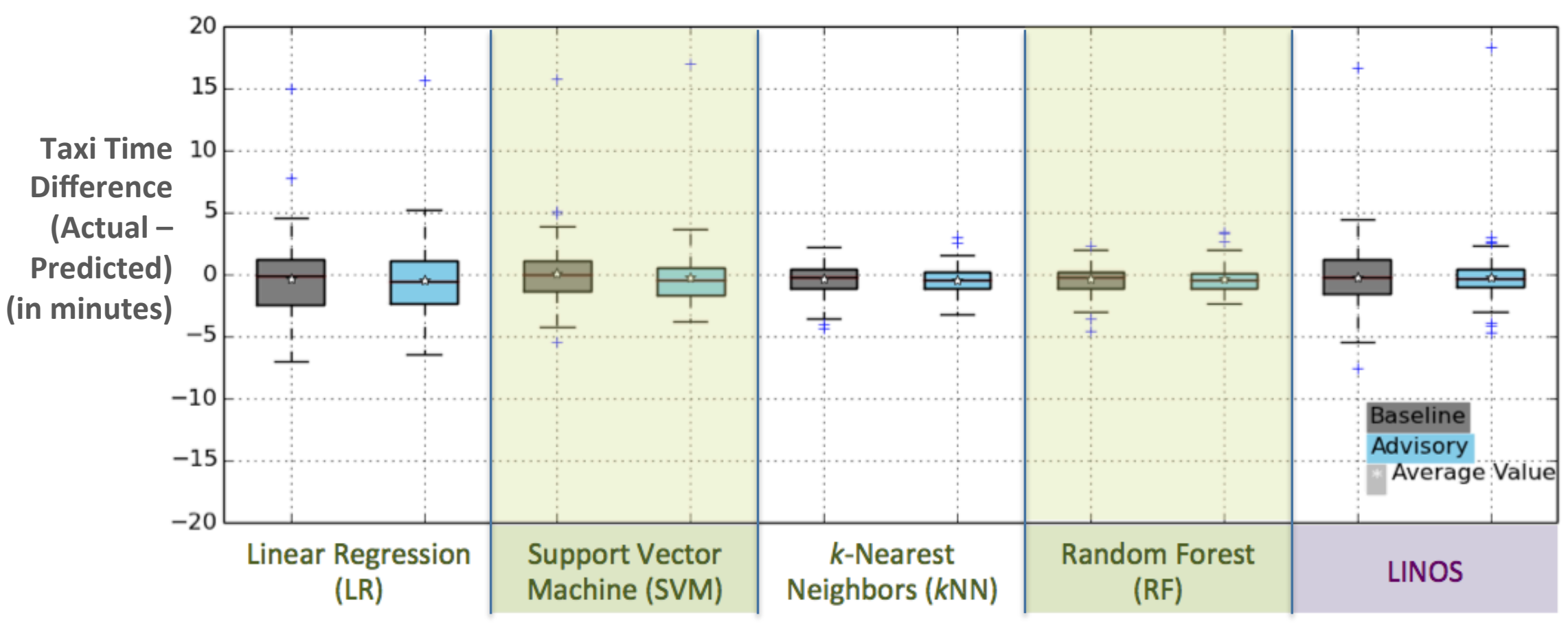




\section{Cumulative Departure Curves with Absolute Prediction Error}

- Except for Linear Regression method, at least $90 \%$ and 97\% departures are within +/- 4-minute error window for Baseline and Advisory runs, respectively.
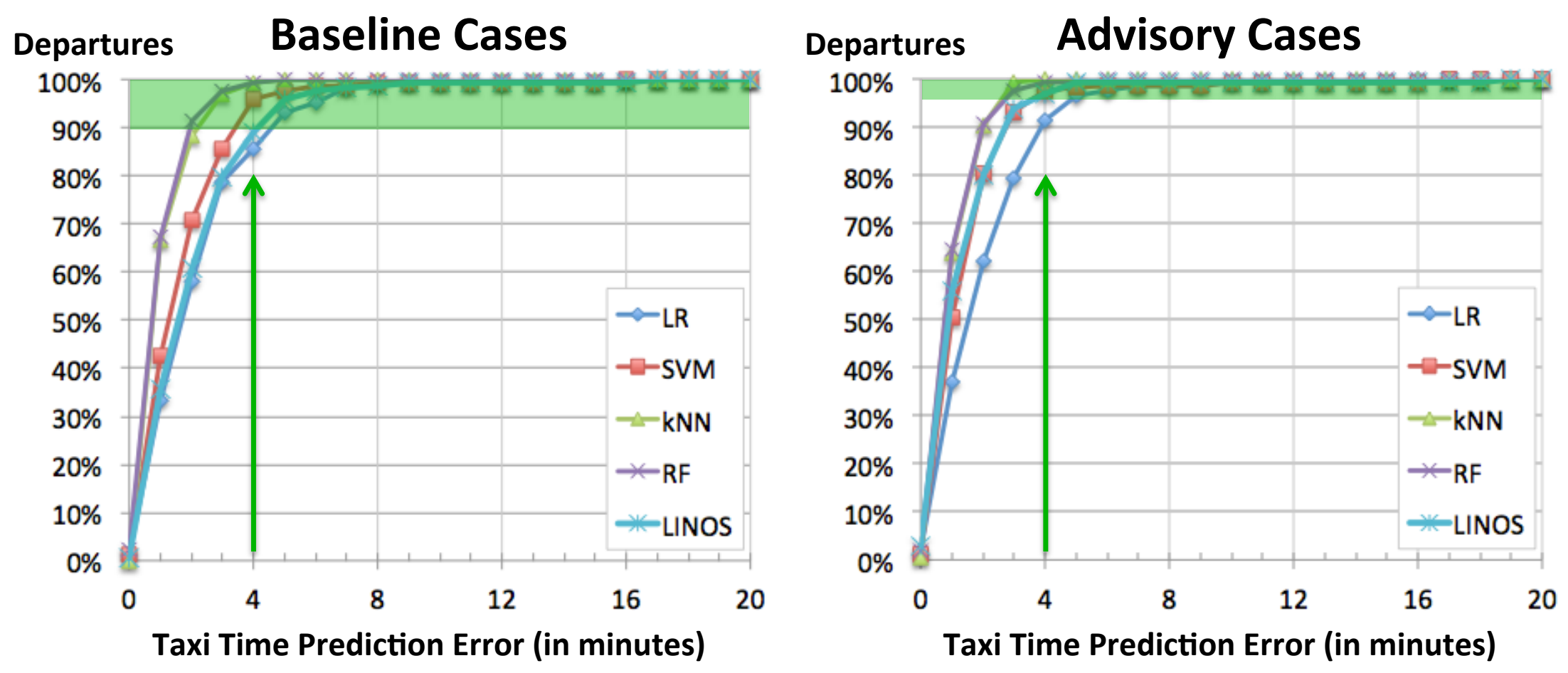


\section{Conclusions}

- LINOS could be used to predict taxi times of departures.

- Showed better prediction accuracy than Linear Regression and Dead Reckoning methods.

- Considered various operational factors, but still needs to be improved.

- M-C routes for arrivals to avoid head-on conflicts

- M-D routes for departures toward Runway $18 \mathrm{~L}$

- Miles-In-Trail (MIT) constraints

- Expect Departure Clearance Time (EDCT) flights (working)

- Intersection takeoffs (working)

- Hardstands in ramp area (working) 


\section{Future Work}

- Apply LINOS to field tests at CLT

- LINOS will be tested in real-time operations.

- Expect better prediction performance by reflecting aircraft status and updating predictions frequently

- Improve machine learning algorithms

- Additional variables from actual traffic data

- Taxi time prediction in real-time operations 


\section{Back-up Slides}




\section{M-C Bypass Taxiway Route}

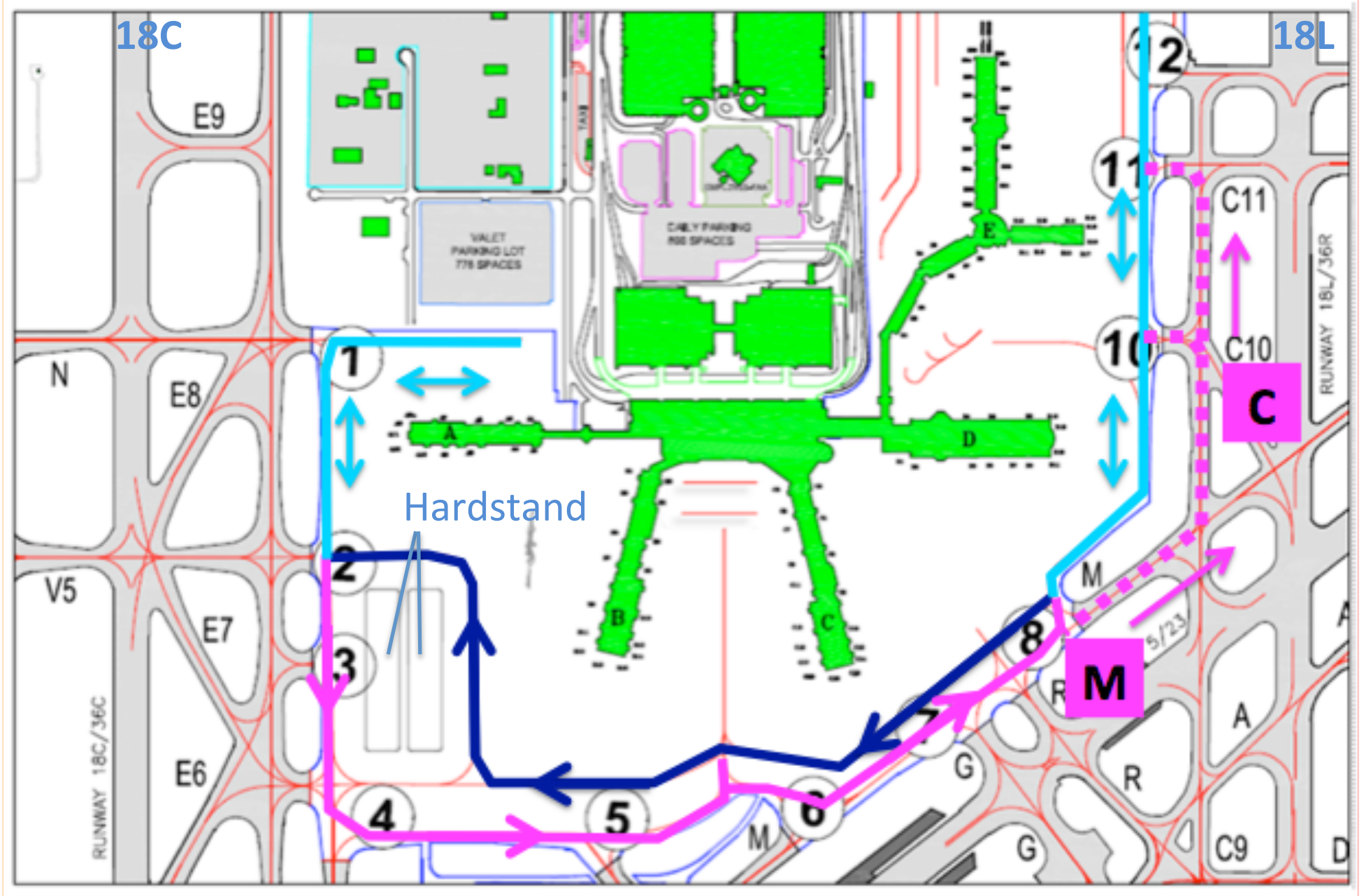

